\title{
Low-level laser therapy for the treatment of androgenetic alopecia in Thai men and women: a 24-week, randomized, double-blind, sham device-controlled trial
}

\author{
Poonkiat Suchonwanit ${ }^{1}$ (D) . Noppanun Chalermroj ${ }^{1} \cdot$ Saranya Khunkhet ${ }^{1}$ \\ Received: 10 September 2018 / Accepted: 28 November 2018 / Published online: 19 December 2018 \\ (C) The Author(s) 2019
}

\begin{abstract}
Low-level laser/light therapy (LLLT) has been increasingly used for promoting hair growth in androgenetic alopecia (AGA). Our institute developed a new home-use LLLT device, RAMACAP, with optimal penetrating energy, aiming to improve therapeutic efficacy and compliance. To evaluate the efficacy and safety of the new helmet-type LLLT device in the treatment of AGA, a 24week, prospective, randomized, double-blind, sham device-controlled clinical trial was conducted. Forty subjects with AGA (20 men and 20 women) were randomized to treat with a laser helmet (RAMACAP) or a sham helmet in the home-based setting for 24 weeks. Hair density, hair diameter, and adverse events were evaluated at baseline and at weeks 8, 16, and 24. Global photographic assessment for hair regrowth after 24 weeks of treatment was performed by investigators and subjects. Thirty-six subjects (19 in the laser group and 17 in the sham group) completed the study. At week 24, the laser helmet was significantly superior to the sham device for increasing hair density and hair diameter $(p=0.002$ and $p=0.009$, respectively) and showed a significantly greater improvement in global photographic assessment by investigators and subjects. Reported side effects included temporary hair shedding and scalp pruritus. In conclusion, the novel helmet-type LLLT device appears to be an effective treatment option for AGA in both male and female patients with minimal adverse effects. However, the limitations of this study are small sample size, no long-term follow-up data, and use of inappropriate sham devices, which do not reflect the true negative control. Trial registration: http://clinicaltrials.in.th/ index.php?tp=regtrials\&menu=trialsearch\&smenu=fulltext\&task=search\&task2=view1\&id=2061, identifier TCTR20160910003.
\end{abstract}

Keywords Low-level laser therapy $\cdot$ Low-level light therapy $\cdot$ Photobiomodulation $\cdot$ Androgenetic alopecia $\cdot$ Female pattern hair loss

\section{Introduction}

Androgenetic alopecia (AGA) is a common hair loss disorder, which affects approximately $80 \%$ and $50 \%$ of Caucasian men and women by age 70 , respectively $[1,2]$. It is characterized by the gradual transformation of terminal to vellus-like hairs resulting in hair thinning with a characteristic pattern. Clinically, men are presented with anterior hairline recession and hair thinning on the vertex. In women, this disorder, also called female pattern hair loss (FPHL), manifests with diffuse

Poonkiat Suchonwanit poonkiat@hotmail.com

1 Division of Dermatology, Faculty of Medicine, Ramathibodi Hospital, Mahidol University, 270 Rama VI Road, Ratchathewi, Bangkok 10400, Thailand hair thinning, mostly on the mid-scalp, and preservation of the frontal hairline. Patients with AGA commonly report a negative psychological impact on the quality of life [3]. Oral finasteride and topical minoxidil are current medications approved by the Food and Drug Administration (FDA) for treating AGA. These two drugs generally provide a favorable result. However, patients experiencing a poor response or unacceptable side effects are in need of additional treatment options. Low-level laser/light therapy (LLLT) has been increasingly used as an alternative treatment for AGA.

LLLT generally utilizes non-thermal effects of lowintensity light at red or near-infrared wavelengths to alter biological activity in cells, termed photobiomodulation or photobiostimulation. Its beneficial therapeutic effects have been shown in a wide range of medical conditions, including wound healing, nerve regeneration, pain reduction, and hair loss $[4,5]$. Although the precise mechanisms of action are not yet well established, strong evidence suggests that LLLT acts 
on mitochondria leading to an increase of reactive oxygen species (ROS) levels, adenosine triphosphate (ATP) production, and induction of transcription factors, which activate genes and produce proteins useful to the cell [4-7].

Hair growth-stimulating effect of LLLT was accidentally discovered by Endre Mester in 1967. Since then, several studies from animal models to clinical trials have cumulatively supported its benefits with minimal adverse events, establishing a novel treatment option for hair loss $[4,5]$. LLLT appears to stimulate anagen reentry of telogen hair follicles and prolong the duration of anagen phase [8-10]. The modulation of hair cycle reveals an increase of hair density and diameter as well as a decrease of hair shedding, resulting in clinical improvement of alopecias. Even though LLLT has been introduced to treat hair loss for over a decade, many questions remain about the better light source between laser and light-emitting diode (LED), the most effective wavelength, optimal therapeutic regimens, and maintenance regimens, and how to choose the right candidate.

The first LLLT device was cleared for use as a safe modality in the treatment of AGA by the US FDA in 2007 for men and in 2011 for women. After that the market has been flooded with numerous LLLT devices claimed to promote hair growth. However, only a small number of these devices have proved their efficacy and safety in a well-designed randomized controlled study. With regard to LLLT devices used for hair loss, there are three basic categories: hood, brush-based (combs, hairbrushes, or headbands), and hat-based (helmets or caps) systems. These devices use coherent diode lasers, incoherent LEDs, or a combination of both as the source of light in the wavelength range of 650-900 $\mathrm{nm}$. Home-use and hands-free devices are principally designed for improving treatment adherence.

In Thailand, a few portable LLLT devices with high-quality clinical evidence are commercially available. The laser comb and laser headband have parting teeth allowing the laser light to reach the scalp surface, but manual movement is needed to treat an extensive scalp area. The helmet combining laser and LED works hands free. However, the query on scalp absorption is mentioned as its main power comes from LED light, which is not collimated and more divergent than laser light. Therefore, a new model of helmet-type LLLT device was invented and named "RAMACAP" under the collaboration between our institute, the Division of Dermatology, Ramathibodi Hospital, and the National Innovation Agency (NIA), a public organization established by the Royal Thai Government, aiming to provide an easy-to-use device with sufficient energy to reach intended targets, in particular, follicular mitochondria. RAMACAP is a portable laser helmet with full scalp coverage and working entirely hands free. The purpose of this study was to assess its efficacy and safety for the treatment of AGA in both men and women.

\section{Methods}

\section{Study design}

A 24-week, prospective, randomized, double-blind, sham device-controlled clinical trial was conducted at a universitybased hospital (Ramathibodi Hospital, Mahidol University, Bangkok, Thailand). The study protocol was approved by the Mahidol University Institutional Review Board on January 20, 2016 (protocol number 01-59-22; clinicaltrials. in.th identifier: TCTR20160910003). Date of first enrollment was January 26, 2016.

\section{Study participants}

Men aged over 18 years with AGA classified as type III vertex, IV, and V by the Norwood-Hamilton classification, and women aged over 18 years with FPHL classified as type I, II, and III by the Ludwig classification were eligible for the study. The exclusion criteria included use of finasteride or dutasteride within 18 months, use of other topical or systemic drugs with hair growth-promoting properties within 6 months, a history of hair transplantation, and patients with a scalp or systemic disease that may affect hair growth. Prior to participation, each eligible subject must provide a written informed consent.

\section{Intervention}

RAMACAP is a combat helmet-shaped device containing single-mode laser diodes, which emit at the wavelength of $660 \pm 10 \mathrm{~nm}$ (Fig. 1). It consists of three layers: (1) the outer protective layer is made from acrylonitrile butadiene styrene plastic; (2) the middle layer is flexible printed circuit boards with 224 pieces of red laser diodes, arranging into four equal sectors; and (3) the inner layer is made from clear thermoplastic elastomers, in order to protect direct contact between laser diodes and human skin/hair. The biocompatibility of inner layer is verified by tests for in vitro cytotoxicity according to ISO 10993-5 and tests for irritation and skin sensitization according to ISO 10993-10.

The maximum optical power for each laser diode is $5 \mathrm{~mW}$ with pulse wave at $4.2 \mathrm{~Hz}$. As a longer distance from the source leads to a lesser light intensity to transmit and absorb, laser bulbs of this model nearly touch the scalp by wearing. The distribution of laser bulbs is also designed for an even coverage across the scalp with a power density of $3.5 \mathrm{~mW} /$ $\mathrm{cm}^{2}$ on the scalp surface. An illumination time of $19.04 \mathrm{~min}$ is required to deliver a fluence of $4 \mathrm{~J} / \mathrm{cm}^{2}$, which appears to be an appropriate dosage for the purpose of stimulating hair growth. Regarding the light source, laser is selected over LED since laser beams are collimated and less divergent, supposing to have better light transmission to intended targets under the 
Fig. 1 RAMACAP, a helmettype portable LLLT device containing 224 red diode laser bulbs $(660 \mathrm{~nm})$

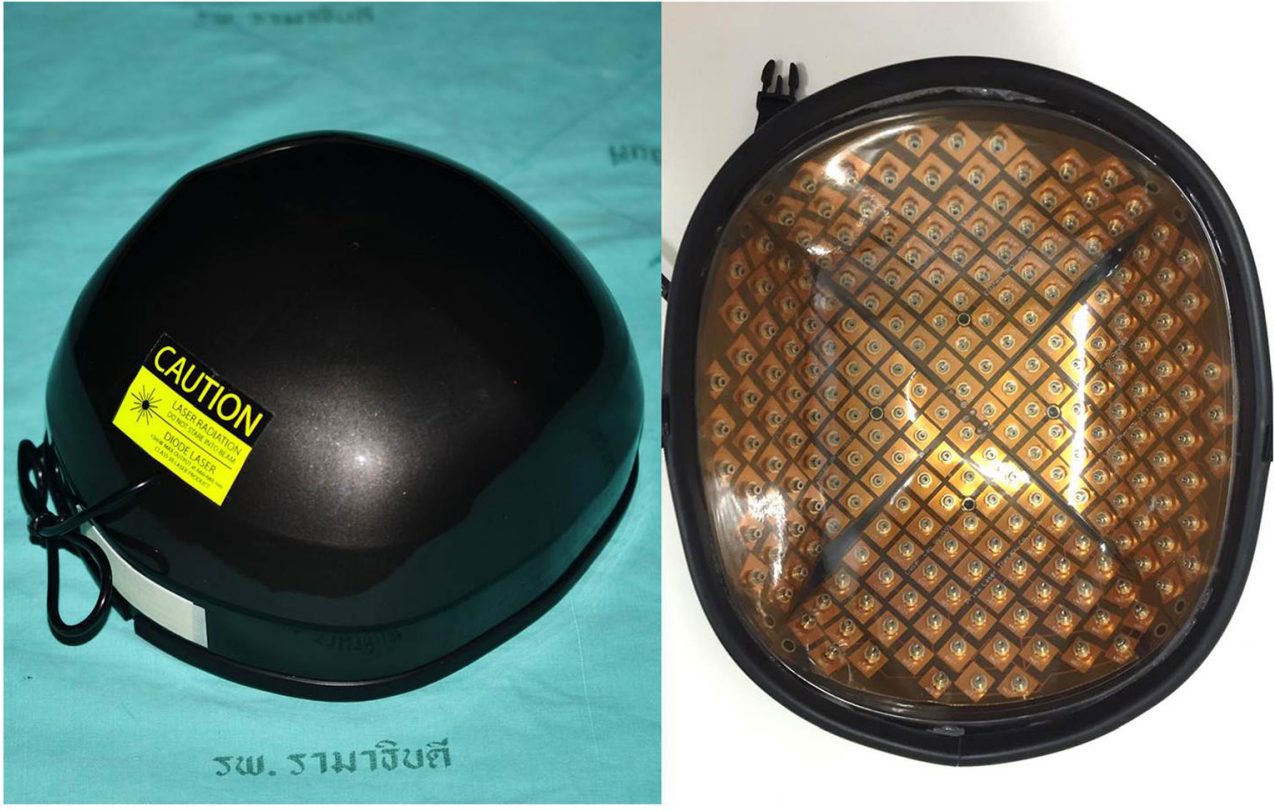

scalp surface. This LLLT system meets FDA requirements for class 3R (IIIa) laser products.

Subjects were divided by using stratified randomization into experimental and control groups. Subjects were firstly stratified by sex and randomly assigned in a 1:1 ratio to use either a laser or a sham device. Laser and sham devices were identical in appearance with the exception of the light source. The sham device contained 224 red LED pods emitting $0.5 \mathrm{~mW}$ of power at the wavelength of $650 \pm 20 \mathrm{~nm}$. Subjects were instructed to use an assigned device at home for $20 \mathrm{~min}$ per session, three times per week, over a 24-week period, as well as to maintain the same hair color, length, and style throughout the study. Investigators and subjects remained blinded to the type of assigned device until study completion.

\section{Assessments}

The primary efficacy endpoint was the change from baseline in hair density and diameter on the target area of the scalp at weeks 8,16 , and 24, photographing with a Folliscope® and measuring with Folliscope 2.8 software (LeadM Corporation, Seoul, Korea). The measurement point was a 1-cm-diameter round area either on the vertex of male subjects or on the midscalp of female subjects, mapped by the intersection point of three distances from fixed anatomical landmarks (tip of the nose, right, and left tragus).

Secondary efficacy endpoints included global photographic assessment (GPA) for hair regrowth by investigators and subjects. Global photographs were taken at baseline and week 24, using a Nikon D5100 DSLR camera (Nikon Corporation, Tokyo, Japan) mounted on the stereotactic head-positioning device for exposure fixation. The photographs were also controlled for camera angles and lighting. Paired photographs were evaluated by a panel of three dermatologists, blinded to study assignment, and the subjects themselves, based on 7 point rating scale $(-3=$ marked deterioration, $-2=$ moderate deterioration, $-1=$ mild deterioration, $0=$ no change, $1=$ mild improvement, 2 = moderate improvement, $3=$ marked improvement). All possible adverse events were recorded at every follow-up visit.

\section{Statistical analyses}

The sample size calculation was based on data from the previous LLLT clinical study in the Asian population [13]. The mean change from baseline to study endpoint in hair density of subjects in the LLLT group was an increase of 17.2 hairs/ $\mathrm{cm}^{2}$ with a standard deviation of 12.1 hairs $/ \mathrm{cm}^{2}$, and those of subjects in the sham control group was a decrease of 2.1 hairs/ $\mathrm{cm}^{2}$. To achieve a power of $80 \%$ and a two-sided significance level of $5 \%$, the minimum sample size required was seven in each group (the experimental group and the control group).

Statistical analysis was performed using SPSS version 18.0 (SPSS Inc., Chicago, IL, USA). The data were not analyzed separately for male and female subjects as the preliminary analysis showed no statistically significant difference in outcomes between genders. Differences in demographic data and global assessment scores were measured using the independent $t$ test or chi-squared test depending on data characteristics. Fleiss's kappa was used to evaluate agreement among three investigators. Changes from baseline in hair density and hair diameter between groups were compared by repeated measures analysis of variance and subsequently analyzed by Fisher's protected least significant difference test. 


\section{Results}

A total of 40 subjects, consisting of 20 men and 20 women, were enrolled and underwent stratified randomization into the experimental group and the control group (10 men and 10 women in each group). Nineteen subjects (10 men and 9 women) in the experimental group and 17 subjects ( 9 men and 8 women) in the control group completed the study. Four subjects withdrew from the study due to personal reasons that were not treatment-related. Analyses for demographic data and efficacy assessments were performed with the intention-to-treat population. A per-protocol analysis was applied for safety evaluation. There were no statistically significant differences between two groups regarding demographics, baseline characteristics, and the severity of hair loss (Table 1).

At week 24 , the laser device was significantly superior to the sham device for increasing hair density and hair diameter ( $p=0.002$ and $p=0.009$, respectively). Laser device-treated subjects showed a significantly greater increase in hair density at weeks 16 and 24 , as well as in hair diameter at week 24 , compared with sham device-treated subjects (all $p<0.05$; Fig. 2). The mean change from baseline to week 24 in hair density was $10.21 \pm 3.25$ hairs $/ \mathrm{cm}^{2}$ in the laser group versus $3.95 \pm 1.32$ hairs $/ \mathrm{cm}^{2}$ in the sham group. The mean change from baseline to week 24 in hair diameter was $6.11 \pm 2.15 \mu \mathrm{m}$ in the laser group versus 3.76 $\pm 1.24 \mu \mathrm{m}$ in the sham group.

Table 1 Demographics and baseline characteristics of the participants

\begin{tabular}{|c|c|c|}
\hline & RAMACAP $(N=20)$ & Sham device $(N=20)$ \\
\hline \multicolumn{3}{|l|}{ Age (years) } \\
\hline Mean (SD) & $38.9(11.3)$ & $35(14.1)$ \\
\hline \multicolumn{3}{|c|}{ Age of onset of hair loss (years) } \\
\hline Mean (SD) & $31.1(11.1)$ & $29(15.3)$ \\
\hline \multicolumn{3}{|c|}{ Duration of hair loss (years) } \\
\hline Mean (SD) & $7.8(3.7)$ & $6(2.2)$ \\
\hline \multicolumn{3}{|l|}{ Classification } \\
\hline \multicolumn{3}{|c|}{ Norwood-Hamilton (10 men), $n(\%)$} \\
\hline III vertex & $3(30)$ & $2(20)$ \\
\hline IV & $5(50)$ & $6(60)$ \\
\hline $\mathrm{V}$ & $2(20)$ & $2(20)$ \\
\hline \multicolumn{3}{|c|}{ Ludwig (10 women), $n(\%)$} \\
\hline I & $4(40)$ & $5(50)$ \\
\hline II & $5(50)$ & $4(40)$ \\
\hline III & $1(10)$ & $1(10)$ \\
\hline \multicolumn{3}{|c|}{ Hair density (hair/ $\mathrm{cm}^{2}$ ) } \\
\hline Mean (SD) & $112.4(18.9)$ & $115.4(16.7)$ \\
\hline \multicolumn{3}{|c|}{ Hair diameter $(\mu \mathrm{m})$} \\
\hline Mean (SD) & 53.8 (11.6) & $54.2(15.1)$ \\
\hline
\end{tabular}
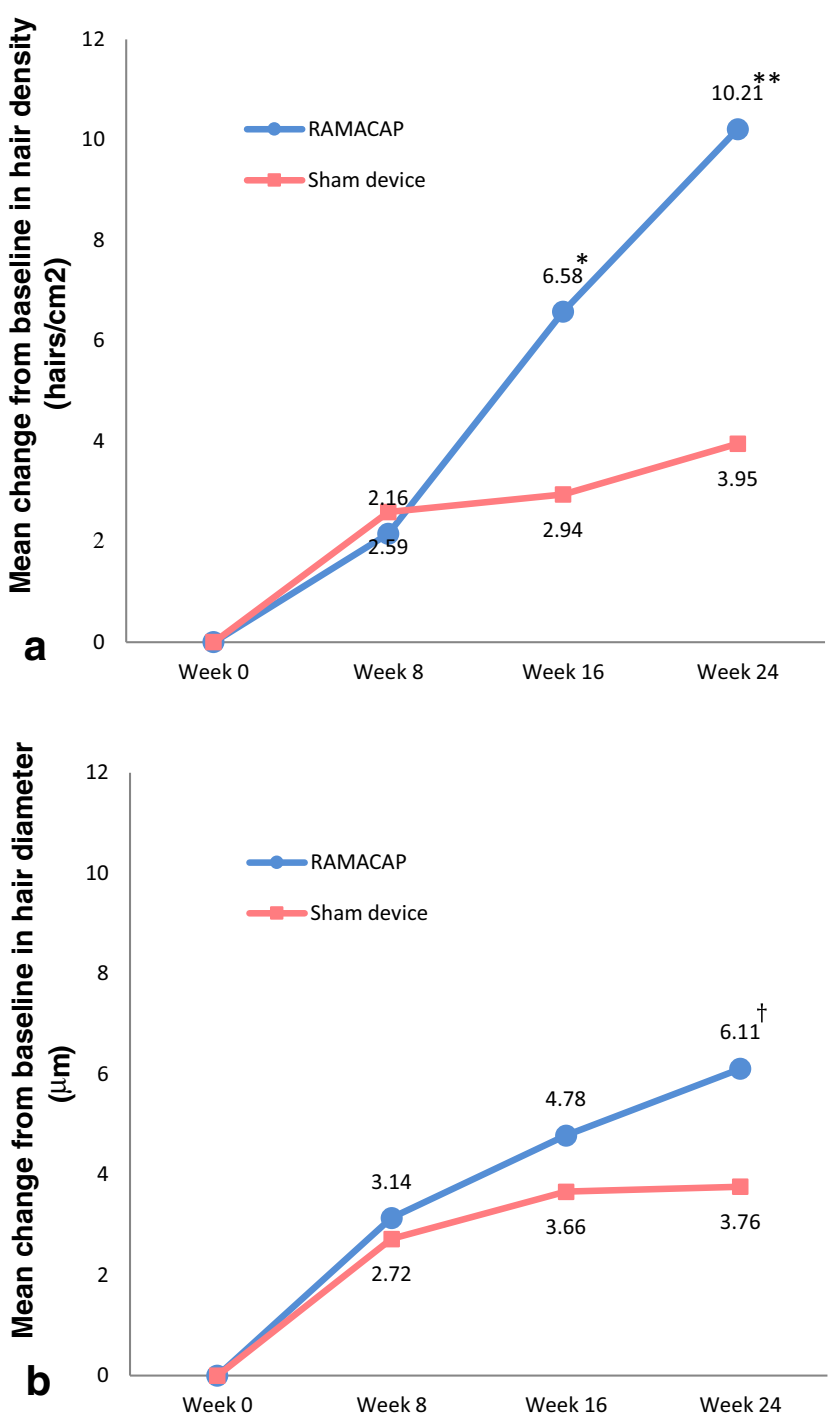

Fig. 2 The mean change from baseline in hair density (a) and hair diameter (b) at weeks 8,16 , and 24 of laser helmet-treated patients and sham device-treated patients. ${ }^{*} p=0.001,{ }^{* *} p=0.002 ;{ }^{\dagger} p=0.009$

Global photographic assessments for hair regrowth after 24 weeks of treatment by investigators and subjects are demonstrated in Fig. 3. Subjects treated with the laser device showed a significantly greater improvement in hair regrowth compared with subjects treated with the sham device for both investigators' evaluation and subjects' self-assessment ( $p=$ 0.0002 and $p=0.0026$, respectively). There was substantial agreement on individual scores rated by a panel of three investigators (Fleiss' kappa $=0.65$ ). Examples of global photographs of laser device-treated subjects, who were rated as having moderate improvement, are shown in Fig. 4.

Incidence of adverse reactions was not significantly different between two groups. There was no serious adverse event reported in any subject. One female subject in the laser group complained of increased hair shedding, which occurred at 2 weeks after starting treatment and spontaneously resolved within 6 weeks. Mild scalp itching was described in two laser- 
Fig. 3 Global photographic assessment based on paired global photographs from baseline to week 24 by investigators (a) and patients (b). Subjects treated with the laser device showed a significantly greater improvement for both evaluations ( $p=0.0002$ and $p=0.0026$, respectively)
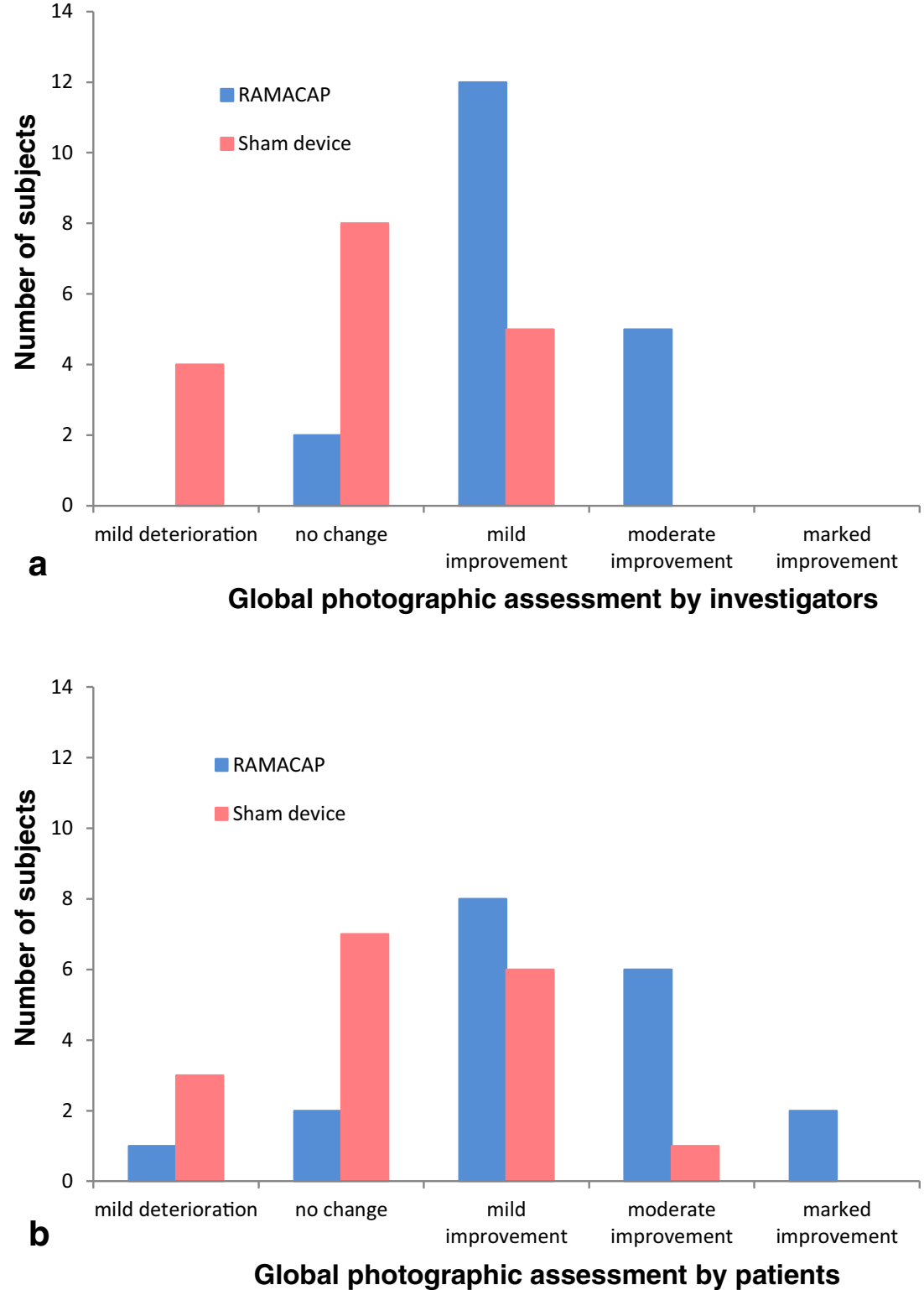

treated subjects and one sham-treated subject without the need for treatment. No subject experienced an adverse event that resulted in the discontinuation or interruption of the study.

\section{Discussion}

LLLT continues to gain popularity in the treatment of AGA due to the ease of use and absence of significant side effects. Apart from anecdotal evidence and non-controlled studies, LLLT showed superior hair growth-promoting effects in men and women with AGA compared to the sham-operating control group in eight randomized controlled clinical trials with the end point varying from 16 to 26 weeks. LLLT devices used in these studies include (1) 655-nm laser combs [11, 12]; (2) a 635-nm and 655-nm laser comb [12]; (3) a helmet using 630-nm LED, 660-nm LED, and 650-nm laser [13]; (4) a helmet using 655-nm LED and 655-nm laser [14, 15]; (5) a helmet using 660-nm LED and 650-nm laser [16]; (6) a 650$\mathrm{nm}$ laser dome fixed in a sport-style hat [17]; and (7) a 655-nm and 808-nm laser scanner [18]. A recent systematic review and network meta-analysis, evaluating the efficacy of nonsurgical treatments for AGA, indicated that LLLT is a highly effective treatment option for AGA; however, the quality of evidence is very low as determined by the risk of bias assessment. Most trials reported funding and support from the device manufacture [19]. High-quality randomized controlled studies are required to confirm this benefit.

The cellular and molecular action mechanisms of LLLT are now much better understood than in the past. Cytochrome $\mathrm{C}$ oxidase (CCO), the terminal enzyme in the mitochondrial respiratory chain, has been most often identified as the primary 


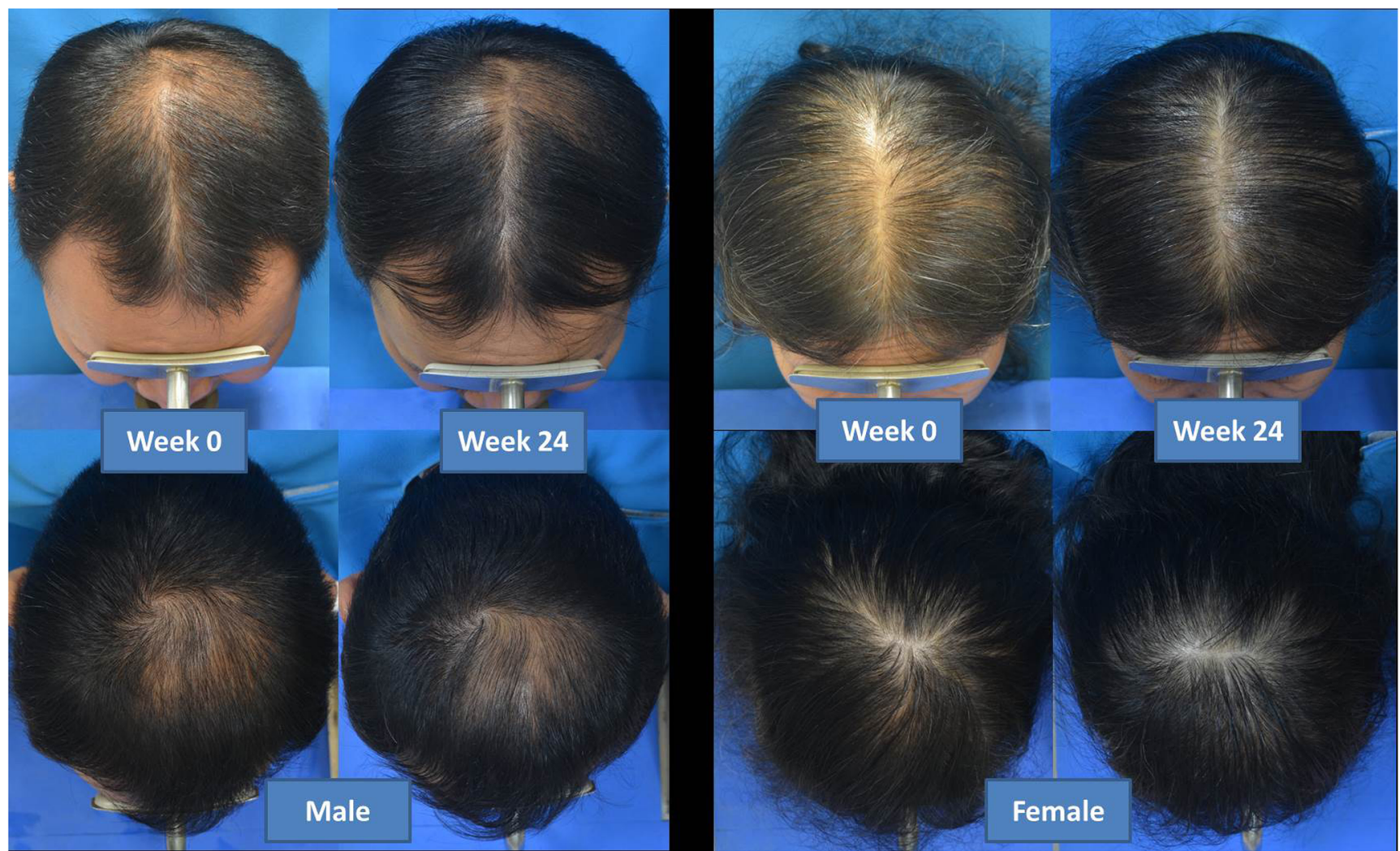

Fig. 4 Baseline and week 24 global photographs of laser helmet-treated patients who were rated as having moderate improvement

photoacceptor or chromophore in LLLT. However, CCO has a weak absorption at wavelengths longer than $900 \mathrm{~nm}$, which suggests the existence of other chromophores. Intracellular water in light-sensitive ion channels, such as the transient receptor potential vanilloid, has been proposed as another candidate chromophore, especially at mid- and far-infrared wavelengths, based on its high absorption capacity in the infrared spectrum [20-22]. The most widely accepted mechanism of LLLT is that nitric oxide, a competitive inhibitor of oxygen binding, is photodissociated from its binding sites in $\mathrm{CCO}$, allowing an immediate influx of oxygen, which drives the electron transport chain to generate ATP and ROS. Afterward, changes in cellular redox state will activate redox-sensitive transcription factor nuclear factor kappa B and activator protein-1. These transcription factors enhance gene transcription and protein synthesis, contributing to cell proliferation and migration $[5,6,22]$.

Regarding LLLT and hair growth-promoting effects, the speculated mechanism mentioned earlier is the direct stimulation of hair follicle stem cells in the bulge region, thereby inducing differentiation and proliferation via increasing the level of heat shock proteins (HSPs), such as HSP27 [4, 9, 23]. Increasing evidence suggests that the transition to anagen phase might be a result of combination effects between (1) direct stimulation on cell proliferation of outer root sheath keratinocytes (ORSKs) and dermal papilla cells (DPCs) and
(2) the release of paracrine growth factors from DPCs, which in turn stimulate proliferation of ORSKs. The ORSK proliferation is upregulated by activating the extracellular signalregulated kinase (ERK) pathway, while the DPC proliferation is stimulated via the $\mathrm{Wnt} / \beta$-catenin and ERK pathways. Several potential paracrine mediators from the dermal papilla induced by LLLT are suggested, hepatocyte growth factor, leptin, vascular endothelial growth factor, fibroblast growth factor 7, lymphoid enhancer-binding factor 1, noggin, Wnt10a, and Wnt10b [5, 10, 24-26].

Biphasic dose response has been demonstrated in a variety of in vitro studies and animal experiments on LLLT [6, 27]. This response follows the Arndt-Schulz rule, which states that for every substance, small doses stimulate, moderate doses inhibit, and large doses kill. Even though convincing instances have not been reported in patients, systematic reviews of randomized controlled clinical trials on LLLT mentioned that some ineffective trials might be explained by overdosing on energy [27]. In addition, effects of LLLT appear to depend on irradiance (or power density; $\mathrm{W} / \mathrm{cm}^{2}$ ) and illumination time rather than fluence (or energy density; $\mathrm{J} / \mathrm{cm}^{2}$ ) [6, 27]. Different irradiances but the same fluence show different results. Insufficient irradiance or too short an illumination time will have no response. Too high irradiance or too long an illumination time will have inhibitory effects. Only an optimal balance between power density and time will produce stimulating 
effects. It is also believed that the absolute figures of this curve are different at different wavelengths and tissue types [27]. Although there is scanty data on the Arndt-Schulz curve in terms of stimulating hair growth, light within the red spectrum at doses of 2 to $4 \mathrm{~J} / \mathrm{cm}^{2}$ is suspected to be appropriate, regardless of the light source $[5,27]$. Shukla et al. showed that the exposure of mice to $632.8-\mathrm{nm}$ laser, emitting $5 \mathrm{~mW} / \mathrm{cm}^{2}$, at a dose of $1 \mathrm{~J} / \mathrm{cm}^{2}$ led to a significant increase in the number of anagen hair follicles, whereas those at a dose of $5 \mathrm{~J} / \mathrm{cm}^{2}$ led to a significant decrease, compared to the control group [8].

This study demonstrated superiority of RAMACAP, the laser helmet, over the sham device by significantly increasing in hair density after 16 weeks and hair diameter after 24 weeks. Analysis of global photographic assessment data also showed a significantly greater improvement in laser helmet-treated patients by both investigator and subject scores. The safety profile was similar to those of devices in the previous clinical trials. There were no serious adverse events reported throughout the study period. One patient in the laser helmet group experienced transient hair shedding during the first 2 months of treatment. Temporary increased hair shedding was also reported in another clinical trial of a 655-nm laser comb. By contrast, it arose in about one third of studied subjects [28]. Increased hair shedding induced by LLLT is most likely related to the synchronization of hair cycle by stimulating telogen hair follicles to reenter the anagen phase, leading to an increase of the exogen phase. An additional adverse effect was mild scalp pruritus, which occurred in both groups. Wearing a helmet might create a warmer environment and higher humidity on the scalp, possibly leading to itchiness.

From the preliminary analysis, when comparing male versus female responses to the laser helmet, the results revealed no statistically significant differences between genders with respect to changes in hair density and diameter, as well as rating scores of GPA for hair regrowth. Likewise, there has been no published article demonstrating a difference in treatment outcomes between male and female patients receiving LLLT for hair loss. This might be explained by the fact that the main mechanism of hair regrowth induced by LLLT is hypothesized to be the stimulation of hair follicle stem cells, ORSKs, and DPCs. The cellular functions of these cells appear to be identical among men and women.

The outcomes of LLLT for hair loss might depend on interindividual differences in hair density, hair length, hair color, and skin color, which are factors affecting absorption and transmission of the light [5]. Long hair, thick layering of hair, dark hair, and dark skin will absorb and reflect more light, resulting in a lower transmission. A bioengineering study investigating the effects of hair color on LLLT demonstrated that light transmission was reduced by $32 \%$ for blond hair and $37 \%$ for black hair [5]. We also observed that subjects who had apparent hair thinning experienced a greater improvement compared to those with milder severity.

In the sham control group undergoing treatment with the 650-nm LED helmet, some patients also showed mild improvement in hair regrowth. This unexpected result could be explained by inadequate light source used in the control group. Hair growth-stimulating effect of LED lights has been previously reported in the literature $[4,10]$. However, since the delivered energy of our sham device was extremely low, therapeutic responses were almost invisible.

The main limitations of this study are the small number of subjects and no long-term follow-up data. A small sample size may reduce the power of the study and increase the margin of error as well as the study subjects may not represent the entire population. The 6-month study duration could not evaluate long-term efficacy and safety. In addition, using a more adequate sham device which yields a true negative effect in the control group could reduce the risk of potential bias in the study. A well-planned large randomized controlled trial with a longer study period is needed to support the benefits and determine whether tachyphylaxis or inhibitory effects could occur. Moreover, studies on optimal therapeutic and maintenance regimens are also essential.

In conclusion, the novel helmet-type LLLT device named RAMACAP might be an effective and well-tolerated treatment option for AGA, at least during the first 6-month treatment period, without age and gender restrictions. The device can be considered as an adjunct to a standard medication or monotherapy for people who refuse to receive pharmacological treatments.

Acknowledgements The authors would like to thank NIA for supporting the experimental materials for use in this study. This study was approved by the MU-IRB (Mahidol University Institutional Review Board) on January 20, 2016 (protocol number 01-59-22).

\section{Compliance with ethical standards}

Conflict of interest The authors declare that they have no conflicts of interest.

Ethical approval This study was conducted in accordance with the principles of the Declaration of Helsinki and in compliance with the International Conference on Harmonization-Good Clinical Practice and local regulatory requirements. The study was reviewed and approved by the appropriate Independent Ethics Committees, and written informed consent was obtained from all subjects prior to study initiation.

OpenAccessThis article is distributed under the terms of the Creative Commons Attribution 4.0 International License (http://creativecommons.org/ licenses/by/4.0/), which permits unrestricted use, distribution, and reproduction in any medium, provided you give appropriate credit to the original author(s) and the source, provide a link to the Creative Commons license, and indicate if changes were made. 
Publisher's Note Springer Nature remains neutral with regard to jurisdictional claims in published maps and institutional affiliations.

\section{References}

1. Severi G, Sinclair R, Hopper JL, English DR, McCredie MR, Boyle P, Giles GG (2003) Androgenetic alopecia in men aged 40-69 years: prevalence and risk factors. Br J Dermatol 149(6):1207-1213

2. Gan DC, Sinclair RD (2005) Prevalence of male and female pattern hair loss in Maryborough. J Investig Dermatol Symp Proc 10(3): 184-189

3. Budd D, Himmelberger D, Rhodes T, Cash TE, Girman CJ (2000) The effects of hair loss in European men: a survey in four countries. Eur J Dermatol 10(2):122-127

4. Avci P, Gupta GK, Clark J, Wikonkal N, Hamblin MR (2014) Lowlevel laser (light) therapy (LLLT) for treatment of hair loss. Lasers Surg Med 46(2):144-151

5. Gupta AK, Foley KA (2017) A critical assessment of the evidence for low-level laser therapy in the treatment of hair loss. Dermatol Surg 43(2): 188-197

6. Chung H, Dai T, Sharma SK, Huang YY, Carroll JD, Hamblin MR (2012) The nuts and bolts of low-level laser (light) therapy. Ann Biomed Eng 40(2):516-533

7. Karu T (1999) Primary and secondary mechanisms of action of visible to near-IR radiation on cells. J Photochem Photobiol B 49(1): $1-17$

8. Shukla S, Sahu K, Verma Y, Rao KD, Dube A, Gupta PK (2010) Effect of helium-neon laser irradiation on hair follicle growth cycle of Swiss albino mice. Skin Pharmacol Physiol 23(2):79-85

9. Wikramanayake TC, Rodriguez R, Choudhary S, Mauro LM, Nouri K, Schachner LA, Jimenez JJ (2012) Effects of the Lexington LaserComb on hair regrowth in the $\mathrm{C} 3 \mathrm{H} / \mathrm{HeJ}$ mouse model of alopecia areata. Lasers Med Sci 27(2):431-436

10. Sheen YS, Fan SM, Chan CC, Wu YF, Jee SH, Lin SJ (2015) Visible red light enhances physiological anagen entry in vivo and has direct and indirect stimulative effects in vitro. Lasers Surg Med 47(1):50-59

11. Leavitt M, Charles G, Heyman E, Michaels D (2009) HairMax LaserComb laser phototherapy device in the treatment of male androgenetic alopecia: a randomized, double-blind, sham device-controlled, multicentre trial. Clin Drug Investig 29(5):283-292

12. Jimenez JJ, Wikramanayake TC, Bergfeld W, Hordinsky M, Hickman JG, Hamblin MR, Schachner LA (2014) Efficacy and safety of a low-level laser device in the treatment of male and female pattern hair loss: a multicenter, randomized, sham devicecontrolled, double-blind study. Am J Clin Dermatol 15(2):115-127

13. Kim H, Choi JW, Kim JY, Shin JW, Lee SJ, Huh CH (2013) Lowlevel light therapy for androgenetic alopecia: a 24-week, randomized, double-blind, sham device-controlled multicenter trial. Dermatol Surg 39(8):1177-1183

14. Lanzafame RJ, Blanche RR, Bodian AB, Chiacchierini RP, Fernandez-Obregon A, Kazmirek ER (2013) The growth of human scalp hair mediated by visible red light laser and LED sources in males. Lasers Surg Med 45(8):487-495

15. Lanzafame RJ, Blanche RR, Chiacchierini RP, Kazmirek ER, Sklar JA (2014) The growth of human scalp hair in females using visible red light laser and LED sources. Lasers Surg Med 46(8):601-607

16. Mai-Yi Fan S, Cheng YP, Lee MY, Lin SJ, Chiu HY (2018) Efficacy and safety of a low-level light therapy for Androgenetic alopecia: a 24-week, randomized, double-blind, self-comparison, Sham Device-Controlled Trial. Dermatol Surg. https://doi.org/10. 1097/dss.0000000000001577

17. Friedman S, Schnoor P (2017) Novel approach to treating Androgenetic alopecia in females with Photobiomodulation (lowlevel laser therapy). Dermatol Surg 43(6):856-867

18. Barikbin B, Khodamrdi Z, Kholoosi L, Akhgri MR, Haj Abbasi M, Hajabbasi M, Razzaghi Z, Akbarpour S (2017) Comparison of the effects of $665 \mathrm{~nm}$ low level diode Laser Hat versus and a combination of $665 \mathrm{~nm}$ and $808 \mathrm{~nm}$ low level diode Laser Scanner of hair growth in androgenic alopecia. J Cosmet Laser Ther. https://doi. org/10.1080/14764172.2017.1326609

19. Gupta AK, Mays RR, Dotzert MS, Versteeg SG, Shear NH, Piguet V (2018) Efficacy of non-surgical treatments for androgenetic alopecia: a systematic review and network meta-analysis. J Eur Acad Dermatol Venereol. https://doi.org/10.1111/jdv.15081

20. Tsai SR, Hamblin MR (2017) Biological effects and medical applications of infrared radiation. J Photochem Photobiol B 170:197207

21. Migliario M, Sabbatini M, Mortellaro C, Renò F (2018) Near infrared low-level laser therapy and cell proliferation: the emerging role of redox sensitive signal transduction pathways. J Biophotonics 11(11):e201700025

22. De Freitas LF, Hamblin MR (2016) Proposed mechanisms of photobiomodulation or low-level light therapy. IEEE J Sel Top Quantum Electron 22(3):348-364

23. Bouzari N, Firooz AR (2006) Lasers may induce terminal hair growth. Dermatol Surg 32(3):460

24. Avram MR, Leonard RT Jr, Epstein ES, Williams JL, Bauman AJ (2007) The current role of laser/light sources in the treatment of male and female pattern hair loss. J Cosmet Laser Ther 9(1):27-28

25. Fushimi $\mathrm{T}$, Inui $\mathrm{S}$, Ogasawara $\mathrm{M}$, Nakajima $\mathrm{T}$, Hosokawa $\mathrm{K}$, Itami S (2011) Narrow-band red LED light promotes mouse hair growth through paracrine growth factors from dermal papilla. J Dermatol Sci 64(3):246-248

26. Joo HJ, Jeong KH, Kim JE, Kang H (2017) Various wavelengths of light-emitting diode light regulate the proliferation of human dermal papilla cells and hair follicles via $\mathrm{Wnt} / \beta$-catenin and the extracellular signal-regulated kinase pathways. Ann Dermatol 29(6):747754

27. Huang YY, Sharma SK, Carroll J, Hamblin MR (2011) Biphasic dose response in low level light therapy - an update. Dose-Response 9(4):602-618

28. Satino JL, Markou M (2003) Hair regrowth and increased hair tensile strength using the Hairmax Lasercomb for low-level laser therapy. Int J Cosmet Surg Aesthetic Dermatol 5(2):113-117 